Journal of Measurements, Electronics, Communications, and Systems (2015) SP0115-02
www.jmecs.org/volla

\title{
Audio Steganography using Modified Enhanced Least Significant Bit in
} 802.11n

\author{
C. F. S. Hartoko ${ }^{1}$, S. Tjondronegoro ${ }^{2}$ and B. Hidayat ${ }^{1}$ \\ ${ }^{1}$ School of Electrical Engineering, Telkom University, Bandung, 40257, Indonesia \\ ${ }^{2}$ School of Electrical Engineering and Informatics, Institut Teknologi Bandung, Bandung, 40116, Indonesia
}

\begin{abstract}
Steganography is a technique to improve the security of data, by inserting messages or confidential information using a medium called the host or carrier or cover. A wide variety of digital media can be used as a host, among others audio, image, video, text, header, IP datagram, and so forth. For audio steganography, the embedded audio is called stego-audio. Steganography can be cracked by using steganalysis. By exploit the weaknesses of each steganography method. Many steganography methods has been developed to increase its performance. This work proposed audio steganography scheme called Modified Enhanced Least Significant Bit (MELSB) which is modified version of Enhanced Least Significant Bit (ELSB). This work use Modified Bit Selection Rule to increase SNR and robustness of stego-audio. After applying MELSB scheme SNR of stego-audio is increased due to its Modified Bit Selection Rule. MELSB scheme also increase robustness of stego-audio. MELSB still works fine until amplification level 1.07. MELSB also works against noise addition better than ELSB and LSB. It gives BER and CER with value 0 at SNR $33 \mathrm{~dB}$. MELSB work fine in real-time condition on 802.11n WLAN if there is no transcoding and noise addition between sender's and recipient's computer.
\end{abstract}

Keywords: 802.11n; Audio; Steganography; Modified Enhanced Least Significant Bit

\section{Introduction}

Steganography is used to improve the security of data, which is by inserting messages or confidential information using a medium called the host or carrier or cover. A wide variety of digital media can be used as a host, among others audio, image, video, text, header, IP datagram, and so forth. As cryptography that can be solved by cryptanalysis, steganography can be solved by using steganalysis. By exploiting the weaknesses of a steganography method, steganalysis continue to be developed in order to solve steganography for a variety of media and methods of insertion.

For this reason, it is necessary to develop a new steganography method or develop from existing methods. Modified Enhanced Least Significant Bit (MELSB) is a method developed from the Enhanced Least Significant Bit (ELSB) method which is included in the temporal domain insertion method category. The ELSB method is more secure than LSB, but it gives stego-audio lower SNR due to its Bit Selection Rule. The differences that owned by MELSB is the Modified Bit Selection rule making confidential information is not easily detected and gives higher SNR than ELSB. MELSB method will be tested on $802.11 \mathrm{n}$ channel to determine the performance and resistance in wireless channel.

\section{Related Work}

\subsection{Steganography}

Steganography comes from the Greek Steganos, which means "hidden" and graphein which means "to write" [1]. Media used to hide secret messages referred to as the host and confidential information that would embed into the host called a message. Media used as a host or a message in the form of multimedia files such as images, audio, video, or text. Steganography is used to send a very important message so as not to be stolen in transmission or to give a sign to a file that can contain information about copyright or hidden serial number.

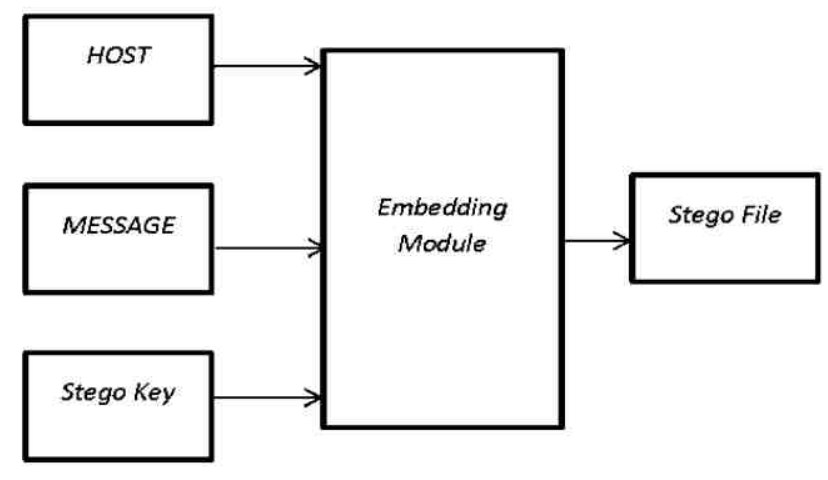

Fig 1. Steganography General Scheme

\subsection{Least Significant Bit Steganography (LSB)}

Least Significant Bit is a method that works by replacing the least significant bit of the cover with a bit message. Examples of the use of this method are described in the following.

0011001110100010101000110010011001011001 0110111010110101000101011110011011011010

Above is a row of bits from the host. Suppose the data that we wanted to embed is a message with a row of bits 1110101011. Rows of the message bits will replace the LSB of the host data. After embedded, a row of bits from the host will be like below 
0011001110100011101000110010011001011001 0110111010110101000101001110011111011011

Advantages of LSB method is easy to implement and high insertion capacity, but this method have a low resistance. Filtration, amplification, and noise addition can easily damage the data [2]. The attacker can easily find a secret message by taking every LSB of each sample.

\subsection{Enhanced Least Significant Bit Steganography (ELSB)}

Enhanced Least Significant Bit [3] is a modification of the method of Least Significant Bit method. This method can be done in two ways. The first way is to randomize the number of bits of the host file that is used for embedding secret messages, this is called bit selection rule. The second way by using selective host sample to embed message bits, this is called sample selection rule. These two rules are used to confuse the intruder and providing more security.

Table 1. Bit Selection Rule [3]

\begin{tabular}{|c|c|c|}
\hline $1^{\text {st }}$ MSB & $2^{\text {nd }}$ MSB & Secret Message Bit \\
\hline 0 & 0 & $3^{\text {rd }}$ LSB \\
\hline 0 & 1 & $2^{\text {nd }}$ LSB \\
\hline 1 & 0 & $1^{\text {st }} L S B$ \\
\hline 1 & 1 & $1^{\text {st }}$ LSB \\
\hline
\end{tabular}

Table 2.Sample Selection Rule [3]

\begin{tabular}{|c|c|c|c|}
\hline $\begin{array}{c}1^{\mathrm{st}} \\
\mathrm{MSB}\end{array}$ & $\begin{array}{c}2^{\text {nd }} \\
\text { MSB }\end{array}$ & $\begin{array}{c}3^{\text {rd }} \\
\text { MSB }\end{array}$ & $\begin{array}{c}\text { Sample } \\
\text { containing next } \\
\text { secret message bit }\end{array}$ \\
\hline 0 & 0 & 0 & $i+1$ \\
\hline 0 & 0 & 1 & $i+2$ \\
\hline 0 & 1 & 0 & $i+3$ \\
\hline 0 & 1 & 1 & $i+4$ \\
\hline 1 & 0 & 0 & $i+5$ \\
\hline 1 & 0 & 1 & $i+6$ \\
\hline 1 & 1 & 0 & $i+7$ \\
\hline 1 & 1 & 1 & $i+8$ \\
\hline
\end{tabular}

\section{Modified Enhanced Least Significant Bit Steganography \\ 3.1 Modified Bit Selection Rule}

Because value with first two MSB " 00 " is smaller than value with first two MSB " 10 " and "11", bit selection rule from ELSB method will be changed.
This is done in order to make changed value on the host are not too visible when message insertion process is done Changes made when 1st MSB and 2nd MSB are 0 and 0 . In ELSB, 3rd LSB is used to embed message bit, but in MELSB when first two MSB is 0 and 0,1 st LSB is used to embed message bit. The sample selection rule in MELSB is still the same as in ELSB

Table 3.Modified Bit Selection Rule

\begin{tabular}{|c|c|c|}
\hline $1^{\text {st }} M S B$ & $2^{\text {nd }}$ MSB & Secret Message Bit \\
\hline 0 & 0 & $1^{\text {st }}$ LSB \\
\hline 0 & 1 & $2^{\text {nd }} L S B$ \\
\hline 1 & 0 & $3^{\text {rd }} L S B$ \\
\hline 1 & 1 & $3^{\text {rd }} L S B$ \\
\hline
\end{tabular}

With combination of modified bit selection rule and sample selection rule, MELSB can provide more security than LSB method and give higher stegoaudio SNR than ELSB. TABLE IV show the comparison between LSB, ELSB, and MELSB insertion method.

Table 4.Comparison of LSB, ELSB, and MELSB insertion method[1]

\begin{tabular}{|c|c|c|}
\hline Method & $\begin{array}{l}\text { Strong } \\
\text { Points }\end{array}$ & Weak Points \\
\hline $\begin{array}{l}\text { Least } \\
\text { Significant } \\
\text { Bit (LSB) }\end{array}$ & $\begin{array}{l}\text { - Low } \\
\text { computatio } \\
\text { nal } \\
\text { complexity } \\
\text { - High bit } \\
\text { rate } \\
\text { - Easier } \\
\text { implementa } \\
\text { tion }\end{array}$ & $\begin{array}{l}\text { Less prone } \\
\text { to attacks } \\
\text { Amplifying, } \\
\text { noise } \\
\text { addition and } \\
\text { compression } \\
\text { of audio will } \\
\text { destroy the } \\
\text { data } \\
\text { Extraction is } \\
\text { easy }\end{array}$ \\
\hline $\begin{array}{l}\text { Enhanced } \\
\text { Least } \\
\text { Significant } \\
\text { Bit (ELSB) } \\
{[3]}\end{array}$ & $\begin{array}{l}\text { Randomness } \\
\text { in the bit } \\
\text { selection and } \\
\text { sample } \\
\text { selection } \\
\text { providing } \\
\text { more } \\
\text { security. }\end{array}$ & $\begin{array}{l}\text { Compression } \\
\text { will destroy the } \\
\text { data }\end{array}$ \\
\hline $\begin{array}{l}\text { Modified } \\
\text { Enhanced } \\
\text { Least } \\
\text { Significant } \\
\text { Bit } \\
\text { (MELSB) }\end{array}$ & $\begin{array}{l}\text { Modified Bit } \\
\text { Selection } \\
\text { give higher } \\
\text { SNR to stego- } \\
\text { audio. }\end{array}$ & $\begin{array}{l}\text { Compression } \\
\text { will destroy the } \\
\text { data }\end{array}$ \\
\hline
\end{tabular}

\subsection{Robustness Test}

This test is conducted to measure MELSB robustness compared with LSB and ELSB method. Bit Error Rate (BER) of inserted message bits and 
Character Error Rate (CER) will be calculated in this test. The message that will be inserted to host audio in robustness test is 'The quick brown fox jumps over a lazy dog 1234567890.' which has total 53 character or 424 bits in 8 bits binary form. Robustness test including:

- SNR Test

This test was conducted to prove that the SNR of audio after embedding messages is larger when using the MELSB scheme (ELSB with modified bits selection rules). This test was conducted using LSB, ELSB, and MELSB scheme. SNR is obtained by using this equation [4].

$$
\mathrm{SNR}=10 \log _{10} \frac{\sum_{0}^{N} \operatorname{Host}(n)^{2}}{\sum_{0}^{N}\left[\operatorname{Stego}(n)^{2}-\operatorname{Host}_{\left.(n)^{2}\right]}\right.}
$$

\section{- Amplification Test}

Amplification test conducted to determine the robustness of the MELSB methods against amplification compared with ELSB and LSB. Amplification here is enlarging the amplitude of the audio signal that had been inserted by a message (stego-audio).

\section{- Noise Addition}

Testing is conducted by add AWGN in the audio signal that had been inserted with a message (stegoaudio) using LSB, ELSB, and MELSB insertion method. SNR of stego-audio continuously increased from $1 \mathrm{~dB}$ to $50 \mathrm{~dB}$.

\section{- Compression / Transcoding Test}

Stego-audio is compressed from $64 \mathrm{Kbps}$ to ADPCM $32 \mathrm{Kbps}$ based on algorithm in [5]. After stego-audio is compressed, message in stego-audio will be extracted, then BER and CER of extracted message is calculated.

\subsection{Implementation in 802.11n}

This method will be implemented in WLAN $801.11 \mathrm{n}$ with 1 spatial stream and $20 \mathrm{MHz}$ channel (MCS 7) in real-time condition. Input host data is speech signal with sampling frequency $8000 \mathrm{~Hz}$ and each sample is encoded to 8 bits. Block diagram of MELSB steganography implementation in $802.11 \mathrm{n}$ is shown in Fig 2. The message that will be inserted into the host is prepared beforehand. Decimal ASCII value of each character is obtained. Then the decimal ASCII value of each character is converted into 8 bits binary number. While user talk through a microphone each voice samples are converted into 8 bits binary form and embedding process will be carried out simultaneously following the pattern in Table 2 and Table 3 . The voice that already contains the message will be sent directly to the recipient without being at the same time. The stego-audio will be send trough TCP/IP. The message that will be inserted into the host is prepared beforehand. Decimal ASCII value of each character is obtained.

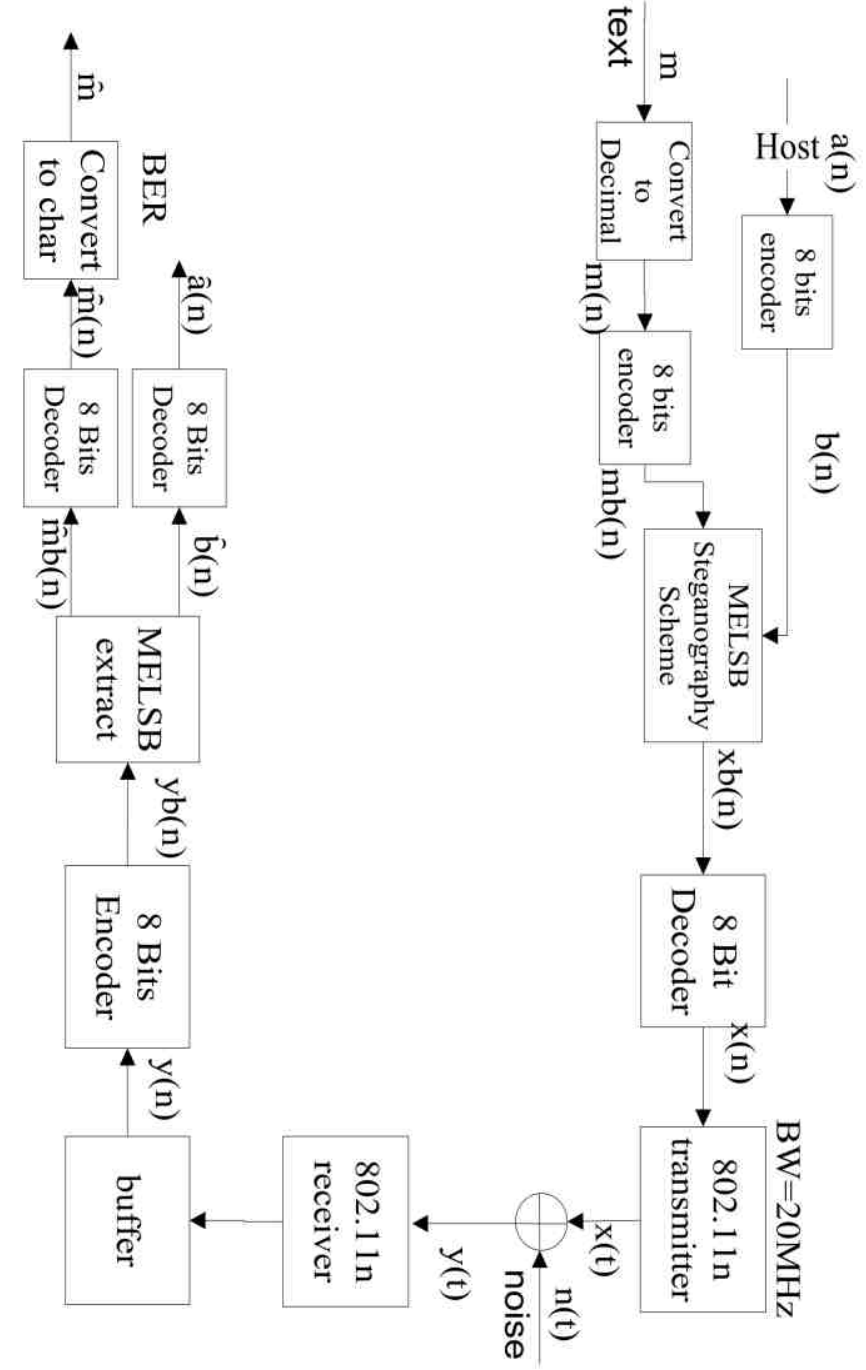

Fig 2. MELSB Steganography Block Diagram in $802.11 n$

Then the decimal ASCII value of each character is converted into 8 bits binary number. While user talk through a microphone each voice samples are converted into 8 bits binary form and embedding process will be carried out simultaneously following the pattern in Table 2 and Table 3. The voice that already contains the message will be sent directly to the recipient without being at the same time. The stego-audio will be send trough TCP/IP. Figure 3 shows the real-time implementation of MELSB in 802.11n Wireless-LAN.

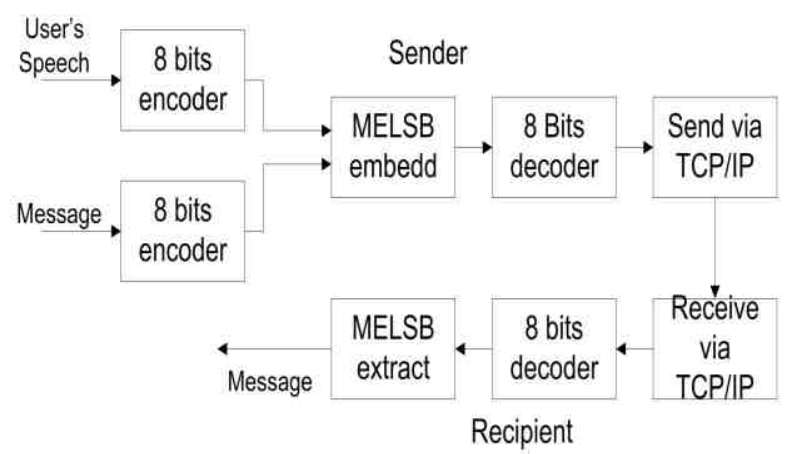

Fig 3. Real-time Implementation Block Diagram 
Hartoko et al. / Journal of Measurements, Electronics, Communications, and Systems (2015) SP0115-02

\section{Robustness Test Result}

This test is conducted to measure MELSB robustness compared with LSB and ELSB method. Bit Error Rate (BER) and Character Error Rate (CER) will be calculated in this test. BER is obtained by comparing extracted message bits before testing with extracted message bits after testing. Figure 4 illustrated how BER is obtained. As seen in figure 4, $1^{\text {st }}$ bit, $3^{\text {th }}$ bit, $4^{\text {th }}$ bit of the message after tested is different with the message bits before tested. After test there are 3 different message bits from total 8 message bits, so the BER is $3 / 8$ or 0.375 .

\begin{tabular}{|c|c|c|c|c|c|c|c|c|}
\hline & 1st bit & 2nd bit & 3rd bit & 4th bit & 5th bit & 6th bit & 7th bit & 8th bit \\
\hline Before test & 1 & 1 & 1 & 0 & 0 & 1 & 0 & 1 \\
\hline After test & 0 & 1 & 0 & 1 & 0 & 1 & 0 & 1 \\
\hline
\end{tabular}

Fig 4. Illustration of Message Bits before Test and after Test

CER is obtained by comparing extracted message characters before testing with extracted message characters after testing. Figure 5 illustrated how CER is obtained. As seen in figure $5,1^{\text {st }}, 2^{\text {nd }}, 3^{\text {rd }}$ and $6^{\text {th }}$ characters of the message after tested is different with the message characters before tested. After test there are 4 different message characters from total 8 message characters, so the CER is $4 / 8$ or 0.5 .

\begin{tabular}{|c|c|c|c|c|c|c|c|c|}
\hline & 1st char & 2nd char & 3rd char & 4th char & 5th char & 6th char & 7th char & 8th char \\
\hline Before test & a & b & c & d & e & f & $g$ & h \\
\hline After test & b & k & s & d & e & c & $g$ & h \\
\hline
\end{tabular}

Fig 5. Illustration of Message Characters before Test and after Test

\subsection{SNR Test Result}

Average SNR after embedding using MELSB scheme is smaller than LSB scheme but larger than ELSB. Modified Bit Selection Rule in MELSB method replace $1^{\text {st }}$ LSB of host-audio when the first two MSB is " 00 ", while Bit Selection Rule in ELSB replace $3^{\text {rd }}$ LSB. So the difference value between host-audio and stego-audio is smaller when using MELSB, with the result that MELSB scheme give larger SNR than ELSB.

\subsection{Amplification Test Result}

MELSB method still works fine until amplification 1.07 , which means amplitude value is enlarged by $7 \%$ from previous value. It means amplification mostly affecting 1st LSB of stego-audio. When the amplitude value of stego-audio is enlarged by amplification 1st LSB of stego-audio is mostly changed.
Table 5.SNR Testing Result Using ELSB, MELSB, and LSB

\begin{tabular}{|c|c|c|c|}
\hline \multirow{2}{*}{ Attempt } & \multicolumn{3}{|c|}{ SNR (dB) } \\
\hline & LSB & ELSB & MELSB \\
\hline 1 & 44.36173 & 35.99525 & 40.63282 \\
\hline 2 & 46.65434 & 33.80618 & 40.15372 \\
\hline 3 & 40.87727 & 36.28362 & 44.21818 \\
\hline 4 & 43.2815 & 37.17845 & 40.86791 \\
\hline 5 & 41.99188 & 34.13527 & 41.3105 \\
\hline 6 & 38.92647 & 33.24726 & 39.17588 \\
\hline 7 & 41.01622 & 33.14977 & 40.84485 \\
\hline 8 & 43.90634 & 37.20582 & 42.06382 \\
\hline 9 & 37.84235 & 34.53976 & 40.69677 \\
\hline 10 & 39.4386 & 35.54426 & 42.48434 \\
\hline 11 & 38.70683 & 37.42117 & 40.53558 \\
\hline 12 & 47.17619 & 39.3466 & 42.74927 \\
\hline 13 & 43.26216 & 33.07516 & 41.107 \\
\hline 14 & 41.38815 & 38.06389 & 38.62263 \\
\hline 15 & 41.50903 & 41.08978 & 38.74961 \\
\hline 16 & 44.02229 & 36.82805 & 44.90617 \\
\hline 17 & 41.01958 & 37.15722 & 39.32776 \\
\hline 18 & 40.61701 & 36.71136 & 41.71994 \\
\hline 19 & 40.16404 & 47.53137 & 42.00134 \\
\hline 20 & 39.40574 & 34.52373 & 42.4399 \\
\hline 21 & 42.13484 & 34.77714 & 42.42505 \\
\hline 22 & 44.81598 & 33.63326 & 39.55485 \\
\hline 23 & 38.88055 & 34.71129 & 40.29103 \\
\hline 24 & 40.32901 & 34.43852 & 42.49332 \\
\hline 25 & 40.51863 & 38.9727 & 39.04027 \\
\hline 26 & 38.34437 & 32.67965 & 41.21652 \\
\hline 27 & 40.05223 & 45.22093 & 44.81666 \\
\hline 28 & 40.73492 & 37.20063 & 42.3634 \\
\hline 29 & 42.29691 & 34.49359 & 39.68386 \\
\hline 30 & 49.53513 & 43.07145 & 45.23205 \\
\hline Average & 41.77368 & 36.73444 & 41.39083 \\
\hline
\end{tabular}

Modified Bit Selection Rule in MELSB have better robustness against amplification, because it replace 3rd LSB when first two MSB is "10" or "11", while ELSB while Bit Selection Rule in ELSB replace 1 st LSB.

\subsection{Noise Addition Test Result}

The test results of noise addition test can be seen in the figure below. All of method work fine if SNR is above $30 \mathrm{~dB}$. 
Hartoko et al. / Journal of Measurements, Electronics, Communications, and Systems (2015) SP0115-02

Table 6.BER after Amplification Testing Result

\begin{tabular}{|c|c|c|c|}
\hline \multirow{2}{*}{ Amplification } & \multicolumn{3}{|c|}{ Message BER } \\
\cline { 2 - 4 } & LSB & ELSB & MELSB \\
\hline 1 & 0 & 0 & 0 \\
\hline 1.01 & 0 & 0 & 0 \\
\hline 1.02 & 0 & 0 & 0 \\
\hline 1.03 & 0 & 0 & 0 \\
\hline 1.04 & 0 & 0 & 0 \\
\hline 1.05 & 0 & 0 & 0 \\
\hline 1.06 & 0.018868 & 0.023585 & 0 \\
\hline 1.07 & 0.044811 & 0.051887 & 0 \\
\hline 1.08 & 0.153302 & 0.134434 & 0.113208 \\
\hline 1.09 & 0.283019 & 0.20283 & 0.113208 \\
\hline 1.1 & 0.384434 & 0.264151 & 0.113208 \\
\hline
\end{tabular}

Table 7.CER after Amplification Testing Result

\begin{tabular}{|c|c|c|c|}
\hline \multirow{2}{*}{ Amplification } & \multicolumn{3}{|c|}{ Message CER } \\
\cline { 2 - 4 } & LSB & ELSB & MELSB \\
\hline 1 & 0 & 0 & 0 \\
\hline 1.01 & 0 & 0 & 0 \\
\hline 1.02 & 0 & 0 & 0 \\
\hline 1.03 & 0 & 0 & 0 \\
\hline 1.04 & 0 & 0 & 0 \\
\hline 1.05 & 0 & 0 & 0 \\
\hline 1.06 & 0.056604 & 0.113208 & 0 \\
\hline 1.07 & 0.056604 & 0.150943 & 0 \\
\hline 1.08 & 0.301887 & 0.245283 & 0.528302 \\
\hline 1.09 & 0.320755 & 0.264151 & 0.528302 \\
\hline 1.1 & 0.566038 & 0.471698 & 0.528302 \\
\hline
\end{tabular}

MELSB produce BER and CER with value 0 at SNR $33 \mathrm{~dB}$, ELSB at SNR $34 \mathrm{~dB}$ and LSB at SNR 35 dB. Sample Selection Rule combined with Modified Bit Selection Rule in MELSB method increase the robustness against noise addition.

\subsection{Compression/Transcoding Test Result}

All of embedding method is weak against compression / transcoding because the amplitude value is changed after compression, so when it encoded into 8 bits binary form, the binary value of amplitude and embedded messages are changed as well.

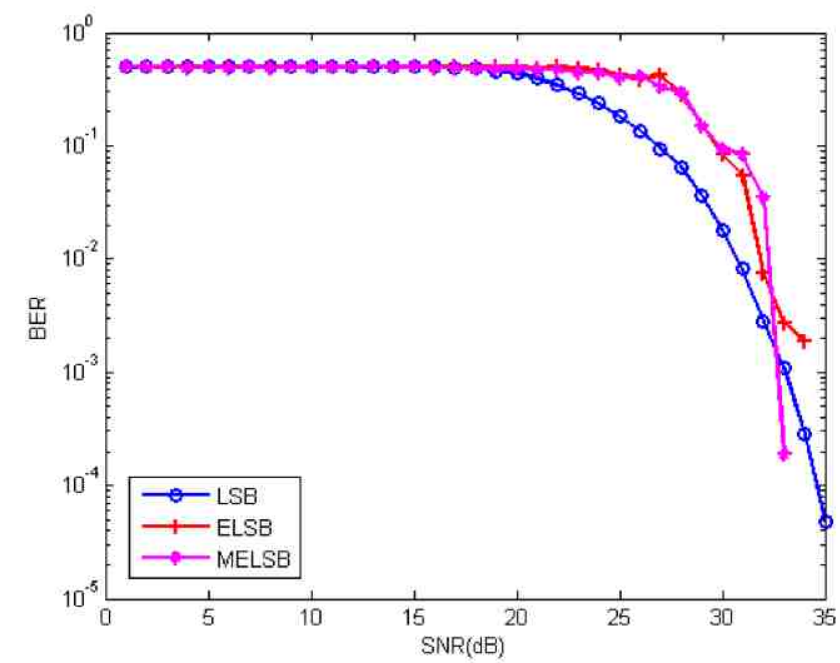

Fig 6. BER Result after Noise Addition

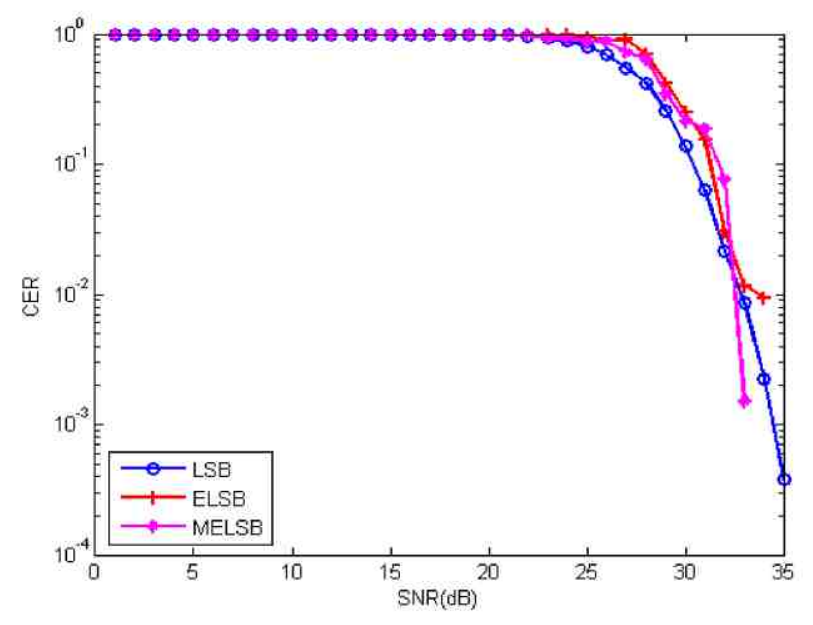

Fig 7. CER Result after Noise Addition

Table 8.Message BER and CER after Compression Result for file 'rekamanlagi.wav'

\begin{tabular}{|c|c|c|c|}
\hline Method & BER & CER & $\begin{array}{c}\text { Extracted } \\
\text { message }\end{array}$ \\
\hline LSB & 0.466981 & 0.981132 & $\wedge \bullet '$ \\
\hline ELSB & 0.436321 & 0.981132 & $"$ \\
\hline MELSB & 0.474057 & 1 & $"$ \\
\hline
\end{tabular}

\section{MELSB Steganography Result In 802.11n WLAN}

MELSB scheme work fine in 802.11n WLAN in real time condition. It gives CER with value 0 , if there's no transcoding and noise addition between sender and recipient. Total average latency in real time condition on $802.11 \mathrm{n}$ is between 900 milliseconds to 1200 milliseconds, while WLAN latency is between 0.8 milliseconds to 5.8 milliseconds. This means MELSB scheme (embedding and extracting process) takes more time be processed. Latency also affected by speech data 
Hartoko et al. / Journal of Measurements, Electronics, Communications, and Systems (2015) SP0115-02

retrieval process from microphone and 8 bit encoder/decoder in sender and recipient side.

Table 9. Steganography Testing Result on $802.11 \mathrm{n}$ Network

\begin{tabular}{|c|c|c|c|}
\hline Place & $\begin{array}{c}\text { DSP } \\
\text { Research } \\
\text { Lab, Telkom } \\
\text { University }\end{array}$ & $\begin{array}{c}\text { B Building, } \\
\text { Telkom } \\
\text { University }\end{array}$ & $\begin{array}{c}\text { Graduate } \\
\text { School } \\
\text { G206, } \\
\text { Telkom } \\
\text { University }\end{array}$ \\
\hline $\begin{array}{c}\text { WLAN } \\
\text { Bit Rate }\end{array}$ & $65 \mathrm{Mbps}$ & $72.2 \mathrm{Mbps}$ & $21 \mathrm{Mbps}$ \\
\hline $\begin{array}{c}\text { Number } \\
\text { of User }\end{array}$ & 49 & 9 & 6 \\
\hline $\begin{array}{c}\text { Sender- } \\
\text { recipient } \\
\text { distance }\end{array}$ & $\pm 3 \mathrm{~m}$ & $\pm 3 \mathrm{~m}$ & $\pm 7 \mathrm{~m}$ \\
\hline $\begin{array}{c}\text { Result } \\
\text { clean, CER }=\end{array}$ & $\begin{array}{c}\text { Voice is } \\
\text { clean, CER } \\
=0\end{array}$ & $\begin{array}{c}\text { Voice is } \\
\text { clean, CER } \\
=0\end{array}$ \\
\hline $\begin{array}{c}\text { Average } \\
\text { total } \\
\begin{array}{c}\text { Latency } \\
\text { (second) }\end{array}\end{array}$ & 0.968906574 & 1.147673246 & 1.149752728 \\
\hline $\begin{array}{c}\text { WLAN } \\
\text { latency } \\
\text { (second) }\end{array}$ & 0.000984625 & 0.000886437 & 0.003047642 \\
\hline
\end{tabular}

\section{Conclusions}

The conclusion that can be obtained from this work is as follows:

a) Modified bit selection rule in MELSB can increase SNR of stego-audio better than ELSB. Because it replace $1^{\text {st }}$ LSB of hostaudio when the first two MSB is " 00 ", while Bit Selection Rule in ELSB replace $3^{\text {rd }}$ LSB. So the difference value between host-audio and stego-audio is smaller when using MELSB, with the result that MELSB scheme give larger SNR than ELSB.

b) MELSB is stronger than ELSB against amplification. Amplification mostly affecting $1^{\text {st }}$ LSB of stego-audio. When the amplitude value of stego-audio is enlarged by amplification $1^{\text {st }}$ LSB of stego-audio is mostly changed .Modified Bit Selection Rule in MELSB have better robustness against amplification, because it replace $3^{\text {rd }}$ LSB when first two MSB is "10" or "11".

c) MELSB is stronger than ELSB and LSB against noise addition due to combination of Sample Selection rule and Modified Bit Selection rule.

d) LSB, ELSB, and MELSB method is cannot work against transcoding. Transcoding changed the amplitude value of stego-audio. When amplitude value is encoded into 8 bits binary form, the binary value of amplitude and embedded messages bits is changed as well

e) MELSB can work fine in real time transmission using $802.11 \mathrm{n}$ Network if there is no transcoding and noise addition between sender and recipient.

f) MELSB scheme takes more times to be processed in real-time condition. As seen in Table 5.9, total average latency is larger than WLAN latency.

\section{Reference}

[1] Jayaram P, Ranganatha H R, Anupama H S. 2011. Information Hiding Using Audio Steganography - A Survey. The International Journal of Multimedia \& Its Applications (IJMA) Vol.3, No. 3

[2] Fatiha Djebbar, Beghdad Ayad Karim, Abed Meraim and Habib Hamam. 2012. " Comparative Study of Digital Audio Steganography Techniques," EURASIP journal on audio, speech and music processing 2012, 2012:25

[3] Muhammad Asad, Junaid Gilani, Adnan Khalid. 2011. An Enhanced Least Significant Bit Modification Technique for Audio Steganography, International Conference on Computer Networks and Information Technology (ICCNIT), pages 143-147

[4] M.Baritha Beguma, Y.Venkataramanib. 2012. LSB Based Audio Steganography Based On Text Compression. International Conference on Communication Technology and System Design. Volume 30, pages 703-710

[5] Richey, Rodger. 1997. Adaptive Differential Pulse Code Modulation using PICmicro $^{\mathrm{TM}}$ Microcontrollers. Microchip Technology Inc.

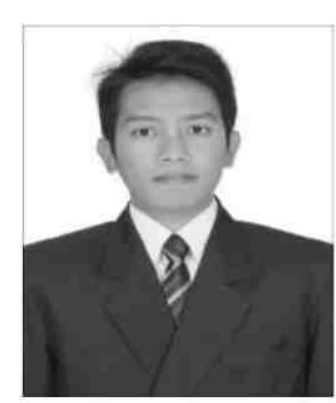

Carolus Ferdy Setiaji Hartoko received the Bachelor degree from School of Electrical Engineering, Telkom University, Bandung Indonesia, in 2014 the Master degree from Master Program of ElectroTelecommunication, Telkum University, Bandung, Indonesia, in 2015. From 2012 to 2014, he was Research Laboratory Assistant at Digital Signal Processing Laboratory, Telkom University. 
Hartoko et al. / Journal of Measurements, Electronics, Communications, and Systems (2015) SP0115-02

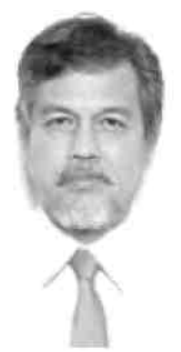

Suhartono Tjondronegoro

received the B.S. degree from Faculty of Electrical Engineering, Institut Teknologi Bandung, Indonesia, the M.S. degree from Electronique, Universite Paul Sabatier-Toulouse III, Montpellier, France, and the Doctoral degree from Electrical Engineering, Academie de Montpellier, Montpellier, France. $\mathrm{He}$ is a lecturer at Department of Informatics and Electrical Engineering, Institut Teknologi Bandung. His research interests include digital signal processing, speech processing, mobile telemedicine system, and wireless communication. He has published a number of papers in international journals and conferences.

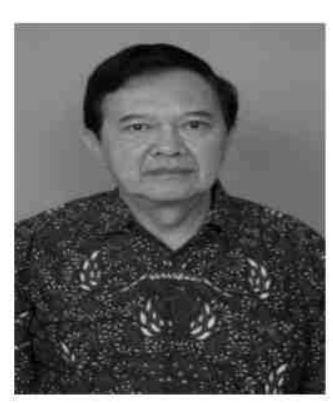

Bambang Hidayat received B.S degree from Faculty of Electrical Engineering, Institut Teknologi Bandung, Indonesia, and the Doctoral degree from Electrical Engineering, Universite de Rennes, France. $\mathrm{He}$ is a lecturer at School of Electrical Engineering, Telkom University, Bandung. His research interests include digital signal processing, speech processing, and image processing. 